\title{
Tabularia
}

\section{Voyages en Russie et définition des marges du monde scandinave dans les fornaldarsögur}

Travel in Russia and the definition of the Scandinavian world's margins in the genre of the fornaldarsögur

Viaggi in Russia e definizione dei confini del mondo scandinavo nelle fornaldarsögur

\section{Benoît Humbert}

\section{OpenEdition}

Journals

Édition électronique

URL : http://journals.openedition.org/tabularia/2236

DOI : $10.4000 /$ tabularia.2236

ISSN : 1630-7364

Éditeur :

CRAHAM - Centre Michel de Boüard, Presses universitaires de Caen

\section{Référence électronique}

Benoît Humbert, « Voyages en Russie et définition des marges du monde scandinave dans les fornaldarsögur ", Tabularia [En ligne], Autour des sagas : manuscrits, transmission et écriture de I'histoire, mis en ligne le 19 mai 2016, consulté le 19 avril 2019. URL : http://journals.openedition.org/ tabularia/2236 ; DOI : 10.4000/tabularia.2236 


\title{
Voyages en Russie et définition des marges du monde scandinave dans les fornaldarsögur \\ Travel in Russia and the definition of the Scandinavian world's margins in the genre of the fornaldarsögur Viaggi in Russia e definizione dei confini
del mondo scandinavo nelle fornaldarsögur
}

\author{
Benoît HUMBERT \\ Doctorant EPHE, CNRS-UMR 8167 Orient et Méditerranée \\ benoithumbert.ephe@gmail.com
}

Résumé:

Thème récurrent de la littérature scandinave médiévale, le voyage en Russie semble évoluer au gré du temps et des genres. La géographie russe s'y présente comme une construction littéraire loin des réalités physiques et historiques, résultat d'un syncrétisme entre différents types de sources. Ce constat est encore plus frappant avec le genre des fornaldarsögur qui figure une véritable évolution où les régions de la Russie médiévale sont pour la première fois décrites, et où la géographie physique fait place à une conception mentale des frontières reposant sur la définition de l'altérité et la prise de conscience de l'unité culturelle scandinave.

Mots-clés: sagas légendaires, Rus' ancienne, monde Viking, altérité, frontières, marges, voyages

\begin{abstract}
:
Recurring theme of medieval Scandinavian literature, travel in Russia seems to evolve according to time and genres. The Russian geography appears in fact as a literary construction far from the physical and historical realities, which results from a syncretism between different types of sources. This is even more striking with the genre of fornaldarsögur which introduces a real change where the regions of Medieval Russia are physically described for the first time, and where the geography gives way to a mental conception of geography and borders based on the definition of otherness and the awareness of a Scandinavian cultural unity.
\end{abstract}

Keywords: legendary sagas, Old Rus', Viking world, otherness, borders, margins, travels

Riassunto:

Tema ricorrente della letteratura scandinava medievale, il viaggio in Russia sembra evolvere a seconda delle epoche e dei generi. La geografia russa si presenta come una costruzione letteraria avulsa dalle realtà fisiche e storiche, risultato di un sincretismo fra diversi tipi di fonti. Questa considerazione è tanto più evidente nel genere delle fornaldarsögur, che mostra un cambiamento significativo: le regioni della Russia medievale sono descritte per la prima volta, e la geografia fisica è sostituita da una concezione delle frontiere basata sulla definizione dell'alterità e sulla presa di coscienza dell'unità culturale scandinava.

Parole-chiave: saghe del tempo antico, antica Russia, mondo vichingo, alterità, frontiere, confini, viaggi 
Dans une Europe médiévale loin d'être figée, où circulent une multitude d'hommes, de marchandises et de savoirs, la littérature scandinave nous offre un grand nombre de témoignages sur ceux qui empruntèrent le chemin menant jusqu'à la Rus' et qui parcoururent les régions de l'Est ${ }^{1}$. Ce trajet n'était pas seulement le fait des Scandinaves vivant en Suède ou en Norvège, mais aussi celui des Islandais et de ceux établis dans les différentes fondations occidentales. Mais avant de poursuivre, il nous importe tout d'abord de définir ce que nous entendons par «voyager en Russie». Cette activité peut en effet revêtir plusieurs caractères - commerce, missions diplomatiques, évangélisme, raids guerriers, migrations - et relève surtout de critères spatio-temporels qu'il nous faut développer. «Voyager en Russie» pour les Scandinaves c'est avant tout emprunter l'Austrvegr, autrement dit la «voie vers l'Est » qui menait de la Baltique à la Rus'.

\section{Les voies de transit dans la Russie médiévale}

De la Baltique, en passant par l'île de Gotland, on pouvait rejoindre le réseau de lacs et de rivières qui parcourait la Russie, la proximité des différentes voies fluviales rendant possible le transfert d'un bassin à l'autre. Ce réseau représentait le point de départ de longues routes commerciales menant jusqu'à la mer Caspienne, la mer Noire, la mer Blanche, ainsi qu'une voie privilégiée pour les différentes migrations. La première de ces routes commençait à partir du golfe de Finlande où il était possible d'emprunter deux voies. La première passait par Ladoga (Aldeigjuborg), puis Novgorod (Hólmgarðr), Gnezdovo, Kiev (Koenugarðr) et débouchait sur la mer Noire et Constantinople. C'est ce que les historiens appellent communément la «Voie des Varègues aux Grecs». Par la Dvina occidentale (Daugava) et ses affluents, il était aussi possible de rejoindre le Dniepr, et d'emprunter cette même route qui menait à Byzance. La seconde partait du golfe de Finlande, via Ladoga, pour rejoindre la Volga puis Bulgar, Itil au nord de la Caspienne, pour ensuite faire route vers Boukhara, Tachkent, Samarkand et Bagdad. Enfin, au nord et au nord-est, il y avait la route qui menait au Bjarmaland en longeant les côtes de la Norvège vers le nord, puis la Laponie et enfin vers la mer Blanche. Le système hydrographique y rendait possible l'accès, à partir de la mer Blanche et de la péninsule de Kola, au lac Ladoga par le Svir, de même qu'au lac Onega à partir de la Dvina du Nord et de ses nombreux affluents.

Cette familiarité avec les régions russes est un phénomène ancien qui remonte aux $\mathrm{VI}^{\mathrm{e}}$-VIII ${ }^{\mathrm{e}}$ siècles, avec déjà des contacts sporadiques aux marges du

1. Sur l'ensemble du corpus historiographique médiéval scandinave qui inclut sagas, documents diplomatiques, vies de saints, inscriptions runiques, traités géographiques et poésie scaldique, 206 voyageurs scandinaves en terre russe sont nommés, dont 96 peuvent être considérés comme des personnages fictifs. Parmi les personnes ayant vraisemblablement existé, 76 sont mentionnées dans 64 inscriptions runiques. Leur recensement est intéressant dans la mesure où ces récits peuvent fournir un regard différent sur ces voyages, et par là même offrir des éclairages sur les raisons plus intimes, plus individuelles de ces entreprises, mais aussi sur le statut et la fonction de ces hommes dans la Rus'. 
nord-ouest de la Russie, motivés par l'exploitation des ressources de la région, avant de prendre à partir du IX ${ }^{\mathrm{e}}$ siècle la forme d'un véritable processus de migration et de colonisation où une aristocratie d'origine scandinave imposa sa domination sur les populations locales, un phénomène qui s'opéra en deux étapes majeures. Ce fut tout d'abord à travers la mise en place d'une entité politique éphémère, le Khaganat russe, centré sur les régions du Volkhov, dont témoignent une demi-douzaine de sources étrangères datées des années 830 à 870 . La plus ancienne, les Annales de Saint-Bertin, décrit ainsi comment une mission byzantine, reçue le 18 mai 839 à Ingelheim à la cour de Louis le Pieux, était accompagnée d'un groupe se réclamant du peuple des Rhôs, que leur roi nommé khagan (chaganus) avait envoyé à l'empereur byzantin Théophile ${ }^{2}$. À travers cette entité, les Scandinaves, peu nombreux, semblèrent entretenir des relations souvent compliquées avec les populations locales, ce qui aboutit à leur «exclusion» autour des années $860^{3}$. La seconde étape fut marquée par un retour durant les années $860-870$ de ce même groupe ethnique d'origine scandinave, les Rus', à l'initiative d'après le Récit des temps passés, des populations du nord-ouest de la Russie qui s'accordèrent pour leur demander de venir les diriger ${ }^{4}$. Ce retour qui s'accompagna d'une acquisition des principaux centres proto-urbains de la région, aboutit à la naissance d'une seconde entité politique, la Rus'. Durant cette période de façonnage de l'État, à cheval sur la période viking, la Rus' continua à entretenir des relations privilégiées avec le monde scandinave, et ce même après que ses élites se sont fondues dans la masse slave au bout de quelques générations. Les Scandinaves continuèrent à affluer vers ces régions, poussés cette fois par diverses motivations: mercenariat, exil, commerce... Les sources russes opèrent d'ailleurs la distinction entre les Rus', ce groupe ethnique à l'origine de l'entité étatique du même nom, et les Varègues qui désignent les Scandinaves venus de l'extérieur, qui parcoururent la terre russe et fréquentèrent les cours princières. De ces deux groupes, les sources écrites scandinaves ne conservent en réalité de traces que du second, bien qu'il faille probablement voir dans certains récits légendaires, une tradition qui refléterait cette colonisation plus ancienne.

\section{Les sources scandinaves}

Parmi les sources scandinaves à notre disposition, nous pouvons distinguer trois genres principaux: les sagas, la poésie scaldique et les inscriptions runiques. Ces sources ont pour avantage le fait qu'elles embrassent directement la période qui nous concerne: runes et stances scaldiques sont datées des $\mathrm{X}^{e}-\mathrm{XI}^{e}$ siècles, tandis que les sagas, rédigées plus tardivement, relatent des faits centrés eux aussi sur la

2. SHEPARD, 1995, p. 41-60

3. Le Récit des temps passés (Povest'vremennykhx let) propose l'année 862, tandis que l'archéologie met en avant des troubles aux alentours des années 865-869. Cette théorie est développée dans ZUCKERMAN, 2000, p. 110-114.

4. Récit des temps passés, entrée 6368-6370/860-862, dans Chronique de Nestor, 2008, p. 50-52. 
période viking. Néanmoins, leurs formes, de même que les informations qu'elles contiennent diffèrent sensiblement l'une de l'autre et ne peuvent ainsi faire l'objet d'un traitement similaire.

Les inscriptions runiques représentent un vaste registre mortuaire agrémenté d'éloges posthumes ${ }^{5}$. Elles sont en cela plus laconiques mais aussi plus précises concernant les faits qu'elles abordent. Elles constituent des marqueurs de ces déplacements et délivrent nombre de détails sur l'identité et parfois même sur le rôle joué par ces hommes.

La poésie scaldique est à peu près toujours strophique et se voit régie par des règles très précises en termes de métrique, d'ordre des mots, de thématiques et de choix de vocabulaire ${ }^{6}$. Les poèmes scaldiques posent cependant un problème en tant que documents historiques car ils ont été transmis plus tardivement par les sagas, et contrairement aux récits dans lesquels ils sont incorporés, les modèles scaldiques, eux, sont contemporains des faits qu'ils évoquent, suggérant ainsi un décalage temporel entre date de création et consignation par écrit. Leur transmission s'est ainsi opérée de manière orale, ce qui laisse supposer des altérations, bien que la rigidité des vers tolère moins de modifications que la prose ${ }^{7}$.

Les sagas sur lesquelles se concentre cette étude représentent de loin notre principale source d'information sur ces déplacements. Ce genre littéraire consiste en un récit en prose dont il existe différentes catégories qui ont existé de manière concomitante. Parmi celles qui concernent directement notre sujet, nous pouvons citer les «sagas royales» ou konungasögur traitant des souverains scandinaves (surtout norvégiens), compilées dans l'ensemble entre 1190 et 1230 ; les «sagas des Islandais» ou Íslendingasögur, rédigées pour la plupart au XIII siècle, qui se rapportent aux premiers Islandais ayant vécu sur l'île aux $\mathrm{X}^{e}$ et $\mathrm{XI}^{\mathrm{e}}$ siècles; les «sagas légendaires» ou fornaldarsögur qui datent de la seconde moitié du XIII siècle et du XIV ${ }^{e}$ siècle, où la part belle est faite aux légendes et au merveilleux ${ }^{8}$. La valeur historique de ces sagas fait encore aujourd'hui débat, du fait de considérations chronologiques, puisqu'elles furent rédigées souvent plusieurs siècles après les événements qu'elles présentent, axés eux sur la période viking ${ }^{9}$. La transmission des faits exposés souffre donc d'un décalage, une transmission orale sur près de deux siècles entraînant très probablement des altérations. L'autre problème réside cette fois dans le sens même que l'auteur souhaite donner à sa saga, le contexte de rédaction pouvant grandement influencer ce dernier. Cela suppose qu'on ne peut considérer ces récits comme "transparents», simples reflets des mondes connus et parcourus. Il faut replacer leur écriture dans un contexte spatio-temporel précis afin d'en dégager à la faveur d'une relecture, l'existence de schémas narratifs spécifiques.

\footnotetext{
5. Marez, 2007.

6. Turville-Petre, 1976.

7. BOYER, 1990.

8. SCHACH, 1993, p. 561.

9. BOYER, 1990; ID., 1984.
} 
Mais de l'étude des figures littéraires et des topoï qui ornent ces récits résulte une interrogation majeure: au-delà de ces constructions littéraires, quel est le réel degré de connaissance des lettrés scandinaves à propos de la Russie médiévale?

\section{La conception de la géographie russe dans la littérature scandinave}

Par les expressions «Russie» ou "espace russe», nous nous référons à l'entité géographique telle que la concevaient les Scandinaves à l'époque médiévale et non telle qu'elle se présentait dans la réalité. Dans les sources scandinaves, à travers l'emploi de toponymes particuliers comme Garðaríki, Austrvegr ou Svipjóð hin mikla ("Grande Suède»), il règne une certaine confusion autour de la notion de Russie qui ne renvoie pas forcément à l'entité politique que représentait la Rus'. Au contraire, la définition de cet espace semble évoluer en fonction des périodes de rédaction et ne saurait figurer un espace aux frontières distinctes. La Russie dans son acceptation géographique figure dans notre cas un espace que les auteurs situaient entre la Baltique à l'ouest, la mer Blanche et la région de Ladoga au nord, Byzance au sud et l'Orient musulman à l'est.

Dans les sources scandinaves, il n'existe pas avant le XIV e siècle de translittération du terme Russie, comme c'est le cas chez les Byzantins sous la forme Rhôsia, ou encore en latin avec les termes Rusia / Ruscia. Étonnamment, elle n'intervient pour la première fois qu'au début du XIV ${ }^{e}$ siècle, sous la forme latinisée Ruzcia, dans l'un des traités géographiques présents dans la Hauksbók, rédigée entre 1302 et 1310, et proviendrait de la traduction d'un terme que l'auteur se permet d'expliquer à son lectorat en précisant: cette Ruzcia, «nous l'appelons Garðaríki» ${ }^{10}$. Les sources antérieures emploient en fait les termes Garðar ou Garðaríki pour désigner la Rus' en tant qu'entité politique ou juste géographique, là où le terme Austrvegr est lui utilisé à propos du voyage vers la Rus' et plus généralement vers l'Est, avant de renvoyer plus tardivement à une entité.

Il existe très peu de descriptions d'itinéraires, c'est-à-dire la description du chemin emprunté et des différentes étapes menant d'un point $\mathrm{A}$ à un point $\mathrm{B}$. Car plutôt que d'envisager la description de ces étapes, les sources scandinaves ont davantage recours à des marqueurs géographiques figurant le déplacement d'un ou plusieurs individus vers une direction. Ainsi, dans la Hákonar saga Hákonarsonar, le Norvégien Ögmundr de Spánheimr a voyagé depuis le Bjarmaland (dans ce cas les côtes de la mer Blanche), vers l'austr («l'est») et les terres de Souzdal, puis encore une fois en direction de l'austr jusqu'à Novgorod et de là, par la route de l'Est (hit eystra), jusqu'à la mer et jusqu'à Jérusalem ${ }^{11}$. Ainsi, les directions indiquées ne correspondent en rien aux réalités géographiques. L'orientation ne se fait donc pas en fonction de notre boussole moderne, mais par rapport à l'espace de destination: quelle que soit la ville à atteindre, du moment qu'elle se trouve au Garðaríki, le voyageur se rendra à

10. Hauksbók, 1892-1896, p. 155.

11. Sturla PórĐarson, «Hákonar saga Hákonarsonar», 1910, p. 371-372. 
l'Est, et poursuivra son chemin en cette direction. Les Scandinaves divisaient en effet le monde en quartiers relatifs aux points cardinaux. L'Est comprenait ainsi la Suède, la Rus', Byzance, la Baltique, parfois le Bjarmaland et les territoires plus à l'est comme le Serkland et d'une manière générale l'Orient musulman ${ }^{12}$. Le Nord se constituait de la Norvège et du Finnmark, l'Ouest des territoires atlantiques - Islande, îles Britanniques, Shetland, Orcades et îles Féroé, France, Espagne, et même l'Afrique - tandis que le Sud correspondait au Danemark, à la Saxe, aux Flandres, mais aussi à Rome. Cette appréhension de la géographie aboutit de fait à une conception singulière des mouvements qui n'est en aucun cas liée à quelque chose de pratique, mais correspond davantage à une vision particulière de l'espace.

L'analyse de l'ensemble des toponymes conservés dans la littérature à propos du voyage vers la Rus' et de la Russie dans son acceptation géographique, nous montre que le spectre d'appréhension des écrivains quant à ces régions était au final très réduit et ne se limitait qu'à un faible nombre de toponymes. À titre d'exemple, seuls quatorze noms de villes ont été conservés parmi lesquels: Hólmgarðr, Aldeigjuborg, Kœnugarðr, Súrdalar, Palteskia, Smaleskia, Móramar, Rostofa, Álaborg, Danparstaðir, Sýrnes, Gaðar, Citopolis et Hélíópólis. Parmi eux, Álaborg, Danparstaðir, Sýrnes et Gaðar (qui pourraient en fait être un seul toponyme $)^{13}$, posent de réels problèmes quant à leur situation géographique, tandis que Citopolis et Héliópólis font figure de villes fictives. Il est ainsi frappant de constater que, comparativement aux toponymes anglais ou nordiques, la littérature scandinave n'a gardé qu'une infime minorité de noms de villes ${ }^{14}$. D'autant que la majeure partie de ces noms de lieux apparaît tardivement par le biais d'une part de traités géographiques rédigés à partir de la seconde moitié du XIII siècle, et d'autre part d'une littérature fantastique qui emprunte énormément à des écrits latins plus anciens, comme ceux d'Isidore de Séville dont sont issus les noms Citopolis et Hélíópólis. Comment dès lors expliquer un tel phénomène? Considérée comme partie intégrante des marges de l'espace scandinave, la géographie russe, n'exigeait probablement pas d'être détaillée.

La Russie des sources scandinaves ne correspond que rarement à la Rus' médiévale telle que l'évoquent les sources slavonnes. Au contraire, elle renvoie à un espace réduit centré autour de quelques grandes villes princières, où se concentraient les activités scandinaves. L’appellatif Garðaríki utilisé pour désigner cet espace résume tout à fait cette idée, puisqu'il renverrait, dans son étymologie, à l'idée d'un royaume dominé par des centres proto-urbains fortifiés ${ }^{15}$. D'autre part, la nature même de la littérature scandinave médiévale, principalement

12. JaCKSON, 2009. Cette «Rose des vents» théorique prendrait ainsi son centre dans le sud de la Norvège, voire au nord de la Germanie. Jackson et Podossinov, 1997.

13. Il pourrait en réalité s'agir du site de Gnezdovo. Джаксон, 1986.

14. À titre de comparaison, ce sont 271 villes de la Rus' qui furent répertoriées dans les chroniques slavonnes (Тихомиров, 1956, p. 42). Коuza (Куза) ajoute à cela 143 villes qui bien que répertoriées dans les strates plus récentes des chroniques, furent en usage durant la période de la Rus' comme l'atteste l'archéologie. Куза, 1975.

15. JACKson, 2003, p. 36; Рыдзевская, 1978, p. 151; sur le terme Garðar voir JACKsON, 2014. 
centrée sur l'action de dirigeants et de dignitaires agissant avec leurs homonymes russes au sein de ces centres politiques et économiques, pourrait aussi être une piste pour comprendre cette absence de descriptions détaillées.

\section{L'évolution des récits de voyage et le genre des fornaldarsögur}

Malgré une fréquentation assidue de voyageurs venus du Nord vers la Rus' et de très nombreux récits traitant de ces derniers, la littérature scandinave ne produit pas de véritable description d'itinéraires et encore moins des régions traversées avant la seconde moitié du XIII ${ }^{\mathrm{e}}$ siècle et la diffusion des fornaldarsögur. Les descriptions les plus anciennes qui appartiennent, dans l'ensemble, aux Íslendingasögur et aux konungasögur, présentent seulement des noms de lieux isolés, avec pour unique ambition de situer avec plus ou moins de précision l'endroit dans lequel se déroule l'action. Les récits à propos de Haraldr harðráði (Haralds saga Sigurðarsonar) ${ }^{16}$, ou d'Óláfr II Haraldsson (Óláfs saga ins helga) ${ }^{17}$, qui sont parmi les plus connus, mais aussi les plus fournis, ne livrent aucune description des itinéraires empruntés, des frontières ni même de la géographie ou de l'ethnographie de cet espace dans lequel les héros sont pourtant amenés à évoluer plusieurs années. Le récit de leurs actions se borne ainsi uniquement aux interactions avec des hauts dignitaires et princes de la Rus', ainsi qu'à de brèves mentions des fonctions qu'ils remplirent dans le cadre de leurs séjours.

Ce type de descriptions connaît par la suite une nette évolution. De simples mentions toponymiques qui situaient le récit dans un espace aux contours flous, on passe à partir de la seconde moitié du XIII ${ }^{\mathrm{e}}$ siècle et le genre des sagas fantastiques, à une véritable volonté de décrire au mieux les territoires dans lesquels se déroule le récit. Parmi ces récits fantastiques qui ont pour cadre la Russie médiévale, nous retrouvons l'Egils saga einhenda ok Ásmundar berserkjabana ${ }^{18}$, la Göngu-Hrólfs saga ${ }^{19}$, la Hálfdanar saga Eysteinssonar ${ }^{20}$, la Hervarar saga ok Heiðreks ${ }^{21}$, l'Örvar-Odds saga ${ }^{22}$, la Völsunga saga ${ }^{23}$, et l'Yngvars saga víðförla ${ }^{24}$.

Toute la nouveauté de ces récits tient dans le fait de placer la terre russe au centre même de l'histoire. Il n'y est plus question de la Rus' en tant qu'État, centrée sur quelques centres urbains, mais d'une terre pleine de mythes et de légendes, tantôt accueillante, tantôt inhospitalière, regorgeant de créatures féroces. Le but de l'auteur n'est plus géographique, mais avant tout littéraire: il ne

16. SNORRi StURLUSON, 1979.

17. SNORri Sturluson, 1983.

18. «Saga d'Egill le Manchot et d'Ásmundr meurtrier des Berserkir», 2012.

19. Saga de Hrólfr sans Terre, 2004.

20. Hálfdanar saga Eysteinssonar, 1917.

21. "Saga de Hervör et du roi Heidrekr», 2012.

22. "Saga d'Oddr aux Flèches", 2012.

23. "Saga des Völsungar», 2012.

24. "Saga d’Yngvar le Grand Voyageur», 2009. 
cherche plus à décrire un lieu de manière avérée, mais à créer une atmosphère globale, un contexte pour l'aventure. D'autant que ces récits semblent présenter un développement et des figures héroïques similaires ${ }^{25}$. Le héros atteint tout d'abord le Garðaríki et sa capitale, où il est accueilli amicalement par les autorités. Il se voit alors confier une quête qui l'amène à entreprendre un voyage encore plus périlleux, qui aboutit généralement à la défaite d'un roi ou d'un ennemi puissant, puis effectue ensuite son retour lors duquel il obtient femme et pouvoir. On retrouve très bien ce schéma dans l'Egils saga einhenda ok Ásmundar berserkjabana, où le héros, Egill, se rend en Garðaríki à la recherche de richesses et de renommée. Après avoir pillé les côtes du pays, il est accueilli par le roi Hertryggr qui le charge de se débarrasser du berserkr Ásmundr qui s'adonne au pillage des régions. Après un duel épique, ils se lient tous deux d'amitié et entrent au service de ce même roi pour retrouver ses deux filles enlevées quelques années plus tôt par des forces mystérieuses, en échange de quoi il s'engage à leur offrir de nombreuses richesses ainsi que le tiers de son royaume ${ }^{26}$. Après s'être rendus jusqu'aux Jötunheimar pour délivrer les deux jeunes filles, ils durent s'allier à une géante et à sa fille en vue d'affronter une horde de ces mêmes créatures, et revinrent ensuite à la cour où, à la place des faveurs promises, ils choisirent d'épouser respectivement Bekkhildr et Brynhildr ${ }^{27}$. À la suite de ces unions, Egill retourna avec sa femme dans le Götaland tandis qu'Ásmundr partira plus à l'est afin d'y explorer la Tartarie.

Ce cheminement du héros, marqué par ces différentes étapes, s'inscrit en fait dans un espace bâti selon un principe de cercles concentriques où quelle que soit la direction, plus il s'éloigne de la capitale, plus les régions deviennent sauvages et dangereuses (figure 1).

Le premier espace correspond toujours au Garðaríki qui se rapporte essentiellement à la région de Novgorod. Remarquons d'ailleurs qu'aucun récit ne mentionne ou ne se déroule à Kiev, pourtant alors la capitale politique de la Rus' durant les périodes où se situent les différentes intrigues. Il s'agit d'un espace pacifié, où les autorités entretiennent des relations très amicales avec les héros. Le second espace est une région peuplée par des communautés humaines, dans laquelle des créatures venues de l'extérieur peuvent cependant évoluer pour y commettre différents forfaits comme l'enlèvement par des géants des filles du roi Hertryggr. Cet espace est contrairement au premier souvent l'objet de conflits, comme dans la Hálfdanar saga Eysteinssonar, où les principaux protagonistes se disputent les villes d'Aldeigjuborg et d'Álaborg ${ }^{28}$. Dans cet espace les populations humaines peuvent aussi se voir affublées de traits monstrueux. C'est le cas des femmes de l'Yngvars saga víðförla qu'Yngvarr décrit comme des «serpents venimeux». Malgré plusieurs avertissements, les femmes de la ville d'Hélíópólis arrivent à s'introduire dans le camp dressé par les Scandinaves répandant la

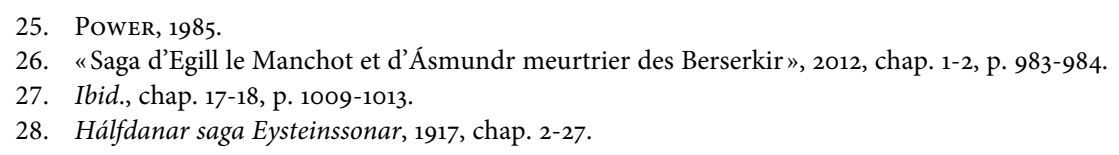




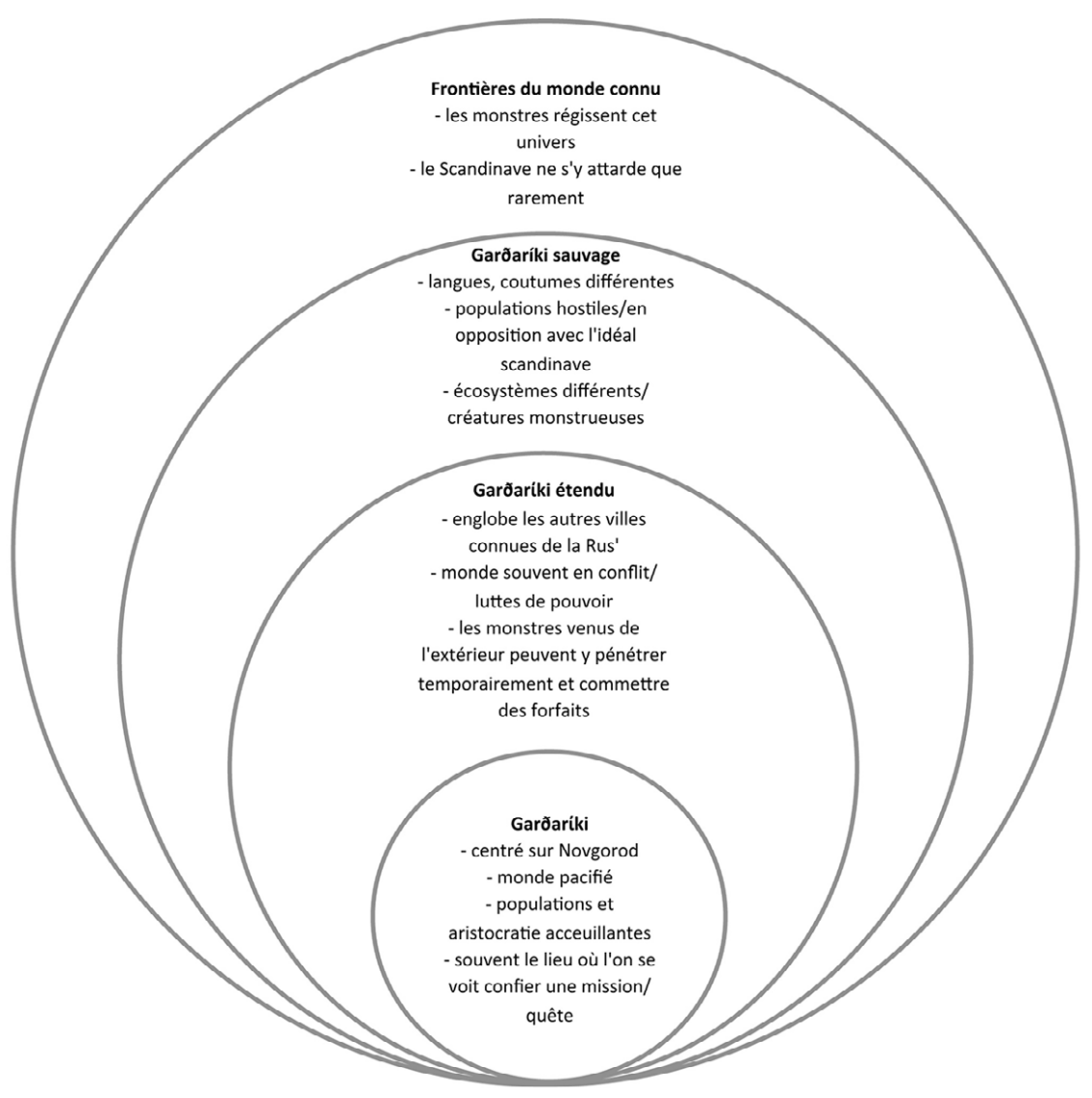

Figure 1 - L'organisation de l'espace russe dans les fornaldarsögur

maladie parmi l'équipage scandinave ${ }^{29}$. La troisième zone est pour sa part peuplée à la fois de communautés humaines et de créatures qui sont hostiles aux héros, et s'en prennent souvent directement à eux. Il peut s'agir comme dans l'Yngvars saga víðförla de géants ou de cyclopes, ou encore de bandits, qui sur des bateaux camouflés en formes de petites îles, attaquèrent l'équipage à l'aide d'une sorte de tuyau d'airain faisant office de lance-flammes, évoquant en quelque sorte le feu grégeois byzantin ${ }^{30}$. Cet épisode n'est donc pas sans rappeler que de nombreux pillards sévissaient sur ces voies de transit. Mais outre ces groupes de bandits, c'est plutôt la réaction des communautés sédentaires qui interpelle, chaque interaction débouchant sur des affrontements nés souvent d'incompréhensions. Alors qu'il faisait commerce avec les populations locales, Sveinn, fils d'Yngvarr «le grand voyageur ", vit un Girskr, c'est-à-dire un habitant venant du Garðaríki, se quereller avec un païen à propos d'un achat de peaux, et le tuer d'un

29. «Saga d'Yngvar le Grand Voyageur», 2009, chap. 7-8, p. 58-59.

30. Ibid., chap. 6, p. 53-54. 
coup d'épée, ce qui déboucha sur une rixe avec les populations locales, Sveinn prenant le parti du «Russe». Peu de temps après, c'est une seconde troupe avec un homme à bec d'oiseau qui s'approcha d'eux en jetant trois pommes, étrange rituel auquel notre héros répliqua par l'attaque ${ }^{31}$. La dernière zone ne voit que rarement l'homme s'y aventurer tant elle semble dangereuse. C'est en cet endroit que se termine la quête et où il s'agit d'affronter de terribles créatures telles que dragons et géants. L'accomplissement de tels exploits apporte alors aux héros richesse et renommée et sonne l'heure du retour.

Le passage d'une zone à l'autre est souvent marqué par un changement de la faune et de la flore qui suscite parfois peur et incompréhension de la part des voyageurs, comme lors de cet épisode amusant où l'équipage de Sveinn ne sut comment réagir à la vue de ce qui pourrait être un éléphant, finissant par exécuter la pauvre bête ${ }^{32}$.

\section{Populations et altérité}

Cette vision particulière de ces espaces où se mêlent mythologie et exotisme reflète en réalité une conception nouvelle du monde et de ses frontières, où le voyage en Russie, une terre pourtant intimement liée à l'histoire des pays nordiques, se présente pour l'auteur comme l'occasion de définir les marges du monde scandinave chrétien par effet d'opposition entre normalité scandinave et altérité russe. En effet, en passant d'un espace à l'autre, le voyageur est amené à côtoyer différents milieux naturels et populations qui s'opposent aux qualités du héros scandinave à la manière d'une sorte de miroir inversé. Malgré quelques détails historiques fiables ${ }^{33}$, la structure littéraire à laquelle répondent ces récits de voyage renvoie en fait à un double impératif: celui de la construction du personnage qui au cours de différentes péripéties acquiert renommée, richesse ou même pouvoir, et celui de l'objectif didactique de l'auteur, qui cherche à faire de ces épisodes autant de récits édifiants porteurs d'une morale précise.

Les protagonistes rencontrés peuvent ainsi être divisés en trois grandes catégories qui véhiculent des idées différentes: la créature fantastique, l'humanoïde et le personnage humain. Cette catégorisation repose sur des principes physiques évidents, mais aussi sur le type de relations que le ou les héros et voyageurs entretiennent avec chacune d'elles. Nous n'utilisons volontairement pas ici l'appellatif «monstre», qu'il nous faut bien distinguer de ces trois sous-ensembles et spécialement de la créature fantastique. L'archétype même

31. Ibid., chap. 9, p. $64-65$

32. Ibid., chap. 9, p. 64-65

33. Un nombre surprenant d'inscriptions runiques atteste la véracité de l'expédition d'Yngvarr sur les routes de l'Est jusqu'à sa disparition aux confins orientaux de la Russie, probablement aux alentours de la mer Caspienne en 1041. Il existe en Suède autour de 25 inscriptions qui font état de l'expédition d'Yngvarr le Grand Voyageur dont la majorité se situe dans la région du lac Mälar. Des annales islandaises confirment quant à elles l'existence d'Yngvarr dont elles datent la mort en 1041. "Saga d'Yngvar le Grand Voyageur», 2009, p. 107-110. 
du monstre représente l'individu ou la créature dont l'apparence, voire le comportement, surprend par son écart avec les normes d'une société, ce qui le pousse ainsi à vivre en dehors de celle-ci. Toutefois, la monstruosité n'est pas forcément négative et encore moins figée: une créature telle que le dragon est supposée agir suivant des procédés qui vont à l'encontre de la société, il en est de même pour certains humains qui de par leurs actes sortent des normes et deviennent dès lors des monstres ${ }^{34}$. La monstruosité est en ce sens étroitement liée à l'altérité et peut s'appliquer dans notre cas aux trois ensembles susnommés. Néanmoins, comment expliquer la présence récurrente de ces créatures dans les différents voyages en terre russe dans le genre des sagas fantastiques? Ce caractère monstrueux produit deux effets distincts: il sert tout d'abord de manière active le récit et magnifie le héros, tandis que d'un point de vue purement symbolique, il participe à la définition de l'altérité et d'un modèle scandinave. Par définition, l'étranger est celui qui n'est pas, celui qui ne possède pas les mêmes qualités, qui ne fait pas partie de la norme et se situe donc en marge d'une communauté. Monstres et étrangers partagent donc cette même situation. Par ce positionnement narratif, en affublant des populations humaines de caractéristiques monstrueuses, l'auteur a donc recours à un procédé d'exclusion de ces populations qui vivent en dehors du monde scandinave et implicitement en dehors du monde chrétien. Ces populations deviennent en quelque sorte les barbares de la Scandinavie.

Contrairement au monstre dans sa nature première, la créature fantastique est une création, consciente ou non, de l'imaginaire humain. Elle rejoint l'idée que le sens commun se fait du monstre: un animal hors normes aussi bien dans son physique que dans son potentiel. Tandis que le monstre est création de la nature et donc soumis à ses lois, la créature fantastique ne connaît aucune limite, ni dans les formes qu'elle peut revêtir, ni dans les prouesses qu'elle peut réaliser. En cela, elle évolue dans un monde sans lois et au rythme de l'imaginaire. Il existe plusieurs types de ces créatures que nous ne pouvons toutes décrire dans cet article. Elles se présentent très souvent comme antagonistes au héros, sur lesquelles une victoire lui permet de parvenir à ses fins. De ces créatures, le dragon est peut-être la plus connue, comme dans l'Yngvars saga víðförla, où lorsque Valdimarr, l'un des membres d'équipage de l'expédition d'Yngvarr, montait la garde de nuit, il fut attiré par la lueur d'un promontoire rocheux couvert de dragons gardant de nombreux trésors. En tentant de s'emparer d'un anneau d'or à l'aide de son épieu, il réveilla un dragon qui éveilla à son tour ses compagnons jusqu'à ce que le plus gros d'entre eux, Jakúlus, soit lui-même tiré de son sommeil. En représailles, ce dernier s'éleva et déversa son venin sur l'équipage de l'un des navires de la flotte, tuant deux prêtres ${ }^{35}$. Le nom de ce dragon semble là encore emprunté aux Étymologies d'Isidore de Séville ${ }^{36}$, ou peut-être

\footnotetext{
34. McLennan, 2010, p. 11-24; JUngmann, 2011, p. 29-42 et p. 59-63.

35. "Saga d'Yngvar le Grand Voyageur", 2009, chap. 5, p. 45-47.

36. The Etymologies of Isidore of Seville, 2006, XI, 3. 16, et XIII. Isidore fait du dragon un être aux caractéristiques serpentines vivant dans les airs et crachant du venin.
} 
à Lucain dans son Histoire des Romains ${ }^{37}$. Il n'est pas ici de notre propos de revenir aux origines du dragon dans la culture de l'Europe occidentale ${ }^{38}$, mais plutôt à travers cet exemple, d'en expliquer la symbolique et d'en saisir la portée dans les descriptions de voyages à l'Est. Bien connu du lectorat latin, dans le contexte des sagas, le dragon présente la particularité d'être le produit d'une union évidente entre mythologie germanique traditionnelle et culture chrétienne. Dans le cadre de cet épisode, sa présence et son action prennent un caractère édifiant et servent d'exemplum: le dragon, personnage maléfique par excellence, punit ceux qui enfreignent les commandements chrétiens. D'autant plus que les membres d'équipages sont à chaque fois prévenus par Yngvarr des risques qu'ils encourent s'ils cèdent à la tentation que représentent sexualité, paganisme ou encore avarice ${ }^{39}$. Yngvarr tout comme son fils plus tard, font figure de saints garants des valeurs chrétiennes par opposition au dragon, créature démoniaque qui tente et châtie le pécheur, et s'oppose ouvertement au christianisme en attaquant les clercs présents dans son équipage ${ }^{40}$. Vaincre le dragon, comme le fera Sveinn au nom de son défunt père, revient ainsi à vaincre le démon et à porter en triomphe les valeurs chrétiennes.

Cette fonction de protecteur des valeurs chrétiennes est à nouveau mise en avant lors de l'épisode où Yngvarr et ses compagnons viennent à bout d'un géant "si terrible qu'ils crurent que c'était le diable » ${ }^{41}$. C'est d'ailleurs grâce aux six jours de jeûne et de prières qu'ils purent accomplir cette prouesse au nom de Dieu, mais aussi de par les qualités de stratège d'Yngvarr. Il remarqua en effet que la maison du géant était soutenue par un unique pilier d'argile qu'il fit affaiblir, pour faire tomber une fois le moment venu la structure sur le géant afin de l'emprisonner et mieux l'achever. Cette attaque n'est pas pour autant gratuite, et semble se justifier par le fait que contrairement aux créatures précédemment rencontrées par le convoi, celui-ci constituait une menace directe pour les humains qu'il se plaisait à enlever pour les attacher à sa ceinture. Par ce combat, Yngvarr s'érige ainsi en protecteur des communautés humaines et ramène la paix dans la région. Ces créatures ne sont pourtant pas toutes menaçantes au premier abord. Au tout début de l'expédition, lors du tour de garde de Garða-Ketill, ce dernier se prit d'envie d'explorer les environs et, une fois arrivé à la fenêtre d'une grande maison où il aperçut une marmite en argent, il s'empressa de la dérober, provoquant la fureur de son propriétaire qui n'était autre qu'un géant ${ }^{42}$. Poursuivi, il abandonna la marmite dont il ne

37. Rómverja saga, 2010, IX, 720.

38. LiONARONS, 1998, p. 1-22.

39. LÖNNROTH, 2012, p. 8-9.

40. Le dragon créature démoniaque opposée au christianisme tire ses origines de l'apocalypse selon saint Jean (12:3 et 12:9) et de l'hagiographie chrétienne où plus d'une quarantaine de saints sont opposés à des dragons, parmi lesquels, saint Michel, saint Siméon le Stylite, saint Clément et bien entendu saint Georges.

41. "Saga d’Yngvar le Grand Voyageur», 2009, chap. 6, p. 52-53. Sur le géant et sa symbolique voir en dernier lieu Schulz, 2004.

42. «Saga d'Yngvar le Grand Voyageur», 2009, chap. 5, p. 45-46. 
conserva que la poignée ${ }^{43}$. Alors qu'il n'avait pas fait preuve d'agressivité, tout comme Jakúlus, le géant n'entra donc dans un état de fureur qu'en réponse au péché commis par le Scandinave. La place accordée à ce type de créatures dans le récit ne revêt pas pour autant uniquement des fonctions édifiantes et ne peut se comprendre qu'en fonction de sa nature profonde, fort différente de celle de la créature fantastique. Il correspond à une créature proche de l'humain dans sa morphologie: bipède, il possède également deux bras ainsi qu'une tête. Dans cette catégorie se mêlent cyclopes, nains, géants ou même encore créatures maléfiques, démons et fantômes qui proviennent d'une véritable fusion entre bestiaire païen hérité de la mythologie scandinave et bestiaire chrétien largement issu de la culture classique ${ }^{44}$. Dans ces récits, elles ne sont en aucun cas considérées comme fantastiques, c'est-à-dire appartenant au domaine du mythe, bien au contraire, elles s'intègrent totalement à la géographie du monde telle que la conçoivent les Scandinaves. Humains et créatures humanoïdes coexistent ainsi, partageant parfois le même type de structures sociales comme dans le cas des géants de l'Örvar-Odds saga qui tiennent conseil auprès de leur chef pour régler leurs problèmes à la manière d'une communauté humaine ${ }^{45}$, ou dans l'Egils saga einhenda ok Ásmundar berserkjabana où ils sont gouvernés par des princes et suivent des coutumes similaires en termes de succession et de mariage ${ }^{46}$.

Comme nous l'avons vu avec la définition de la monstruosité, c'est la nonappartenance à la société et à son ensemble de normes qui définit l'altérité et par là même la monstruosité d'un être vivant. En ce sens, les créatures des sagas dépassent la simple limite existant entre humanité et monstruosité. Ces différences n'étant pas clairement définies, elles permettent l'interaction entre ces deux ensembles, comme dans la Hervarar saga ok Heiðreks, où le roi du Garðaríki, Svafrlami, se fait forger l'épée magique Tyrfing par les nains Dvalinn et Durinn ${ }^{47}$. Ce traitement n'a rien de surprenant lorsque l'on considère le cadre d'écriture et l'influence hautement chrétienne de ces différentes œuvres. On sait que ces créatures humanoïdes rencontrèrent une véritable réflexion au sein de la culture chrétienne du Moyen Âge qui visait à leur trouver une place dans le monde ${ }^{48}$.

43. Garða-Ketill semble d'ailleurs tenir son nom de cet épisode: la première partie de son nom suggère un séjour en Garðar tandis que la seconde renvoie à l'idée d'une bouilloire et de sa poignée si caractéristique.

44. Au Moyen Âge, la littérature autour d'Alexandre le Grand et de son voyage en Inde, de même que les écrits de Pline (Naturalis Historia) et de Solinus (De mirabilibus mundi ou bien Collectanea rerum memorabilium) représentent les principales sources concernant les créatures fantastiques et les monstres.

45. «Saga d'Oddr aux Flèches», 2012, chap. 6, p. 845-846.

46. "Saga d'Egill le Manchot et d'Ásmundr meurtrier des Berserkir», 2012, chap. 9-15, p. 995-1005.

47. "Saga de Hervör et du roi Heidrekr», 2012, chap. 1, p. 120. Durinn est mentionné dans la Völuspá (strophe 10) comme étant le second nain crée après Mótsognir, tandis que Dvalinn apparaît dans le Grímnismál (strophe 14) comme l'un des quatre nains qui se nourrissaient auprès d'Yggdrasill. Enfin, dans l'Hávamál (strophe 143) il est celui qui enseigna les runes à ses congénères. Voir dans «Saga des Völsungar», 2012.

48. SIMEK, 1996, p. 86-97. 
Mais dans le schéma narratif des fornaldarsögur il semble que cela soit différent. Au niveau littéraire, la figure même du héros ne semble s'envisager qu'au travers des aventures qu'il vit et des créatures auxquelles il fait face. Werner Schäfke a très justement remarqué que les héros de ces romans tendaient à effectuer ces voyages en terres lointaines afin de combler certains manques ${ }^{49}$. En l'absence de l'une des valeurs de son espace d'origine (beauté, courtoisie, tempérance, qualités chevaleresques), il doit ainsi quitter ce dernier et s'aventurer dans le monde extérieur peuplé de créatures reflétant l'exact opposé de ses qualités (sorcières, berserkir, géants). Tant qu'il est dans ce monde, le comportement du héros s'adapte alors à son environnement et peut devenir l'équivalent de celui des créatures qui le peuplent, ne répondant plus aux règles du monde civilisé ${ }^{50}$.

Pour leur part, les communautés humaines peuvent être divisées en deux ensembles. Le premier se trouve composé d'aristocrates et de dignitaires qui accueillent presque toujours avec bienveillance les Scandinaves. Cette entrevue avec un grand personnage constitue ainsi très souvent le point de départ d'une quête qui amène le héros plus loin dans les terres, le poussant à côtoyer de nouvelles figures humaines parmi les populations rencontrées sur son trajet. L'attitude de ce second groupe varie grandement selon la distance qui sépare les protagonistes de leur point de départ en fonction des cercles précédemment évoqués. Là encore, l'interaction avec ces populations sert à la fois le développement du héros, mais aussi de récit édifiant comme dans le cas des femmes de l'Yngvars saga víðförla. Malgré les avertissements d'Yngvarr, les femmes de la ville d'Hélíópólis arrivent à s'introduire dans le camp dressé par les Scandinaves. La plus noble d'entre elles parvient alors à se glisser dans la tente du héros, qui n'arrive à repousser ses avances qu'en la poignardant dans les parties intimes. Mais malgré la vertu d'Yngvarr, une maladie s'abat sur l'expédition un peu à la manière d'une punition divine, après que certains de ses compagnons eurent cédé à ces femmes. C'est seulement à la mort d'Yngvarr, bien conscient que sa disparition lèverait la malédiction, que son équipage fut sauvé, à la manière $\mathrm{du}$ Christ se sacrifiant pour racheter le salut des hommes. La femme, dans cet épisode revêt un double caractère: elle fait à la fois figure de personnage sauvage, mais aussi de tentatrice. D'un point de vue symbolique, cet acte ne correspond ni plus ni moins qu'à la victoire du héros sur les péchés de chair et de luxure, magnifiant ainsi les qualités de ce dernier au détriment de celles de son équipage, dont certains membres succombèrent à la tentation, ce qui entraina en l'espace de quelques heures leur mort. Mais par cet appétit sexuel, la femme fait aussi figure de barbare, rappelant notamment les Amazones d'Hérodote, d'autant qu'elles semblent vivre sur le même territoire, c'est-à-dire en Scythie comme le suggère le nom de la ville située plus en amont: Citopolis ${ }^{51}$. La lubricité et la fornication à outrance sont souvent présentées comme caractéristiques du barbare, et se définissent ainsi comme autant d'éléments transgressifs. Les femmes

49. SCHÄFKE, 2009; GlaUSER, 1983.

50. Ibid.

51. Godolphin, 1973-1974, p. 129-149; Brown et Tyrell, 1985. 


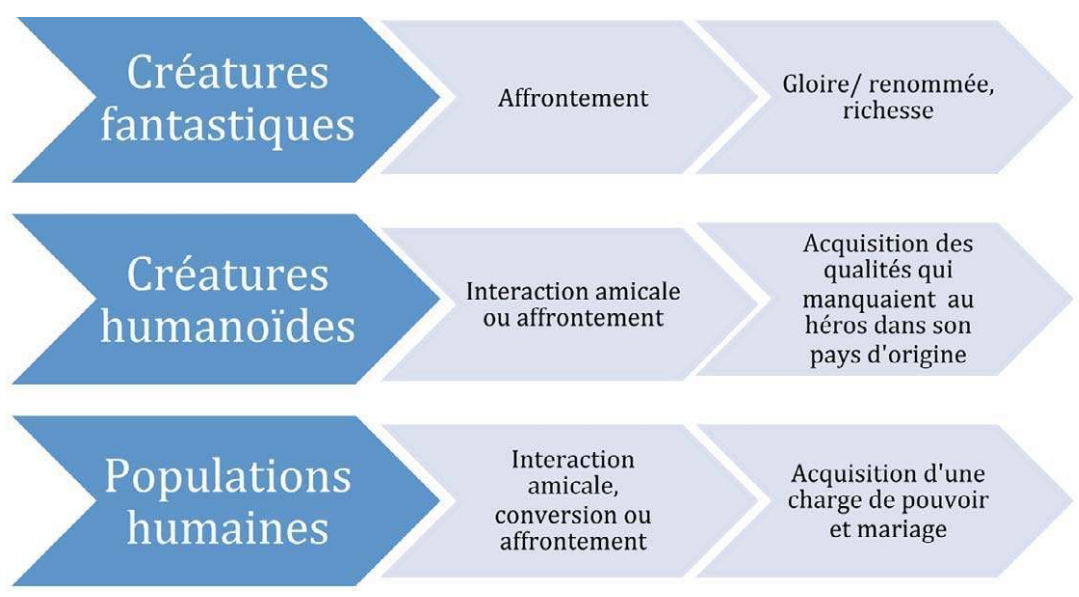

Figure 2 - Évolution du héros au contact des populations et créatures de la Rus'

dans l'Yngvar saga víðförla apparaissent donc comme formant une caste à part, dont il faut se méfier. Or, lorsque l'on considère les rouages de la société et des institutions de l'Islande médiévale dans laquelle a été rédigé ce type d'ouvrages, on se rend compte que la femme, garante du domaine privé, mais exclue de l'Alping et par là même du domaine politique mais aussi religieux, appartient, elle aussi, aux marges de la sociétés ${ }^{2}$. Si l'on ajoute à cela les conceptions chrétiennes assez répandues qui font de la femme la tentatrice originale, on comprend mieux le rôle qu'elle occupe dans ces récits. Cet épisode édifiant, comme ceux énumérés auparavant participent à la construction du personnage d'Yngvarr en tant que saint, qui par écho contribue à la définition d'un modèle humain auquel le pieux lecteur pourra se référer et calquer ses agissements (figure 2).

\section{La définition des marges du monde scandinave}

L'ensemble de ces créatures et populations cohabitent donc aux marges du monde scandinave dans une zone qui n'est pas clairement définie et qui peut couvrir un espace quasiment infini propice à l'imagination des auteurs ${ }^{53}$. Fleuves, montagnes, océans ou encore épaisses forêts servent alors de frontières naturelles et séparent cet «autre monde» du monde des hommes. Mais cette frontière physique qui définit le monde scandinave s'affiche aussi comme une véritable frontière psychologique et culturelle entre le monde connu et l'ailleurs, le monde de l'autre, le monde de l'imaginaire et du surnaturel. Le Garðaríki, divisé en différents espaces, est ainsi perçu malgré sa proximité géographique tel un pays lointain dans les esprits des Scandinaves ${ }^{54}$. Dans les descriptions géographiques

52. Ahola, 2009, p. 21-28.

53. SIMEK, 1986, p. 247-275.

54. Shafer, 2010, p. 7-8. Sur le Garðaríki, voir JACKsON, 1993. 
que nous offrent les auteurs scandinaves à propos de cet espace, il n'est en aucun cas fait mention de distances ${ }^{55}$. Distinction est faite cependant entre les contrées qui sont proches car familières, et celles qui sont éloignées car considérées souvent comme exotiques. Cet espace ne connaît d'ailleurs de frontières que par la rupture qu'il offre avec l'univers scandinave à l'ouest et avec l'Empire byzantin au sud, tandis qu'au nord et à l'est, ses frontières varient selon la plume des auteurs, pour qui le changement de milieux naturels constitue les marqueurs essentiels du passage d'une région à l'autre ${ }^{56}$. Mais ces descriptions de la Russie médiévale, de ses paysages, de ses populations, et des interactions entre héros et populations locales, participent en fait à deux phénomènes étroitement liés: celui du développement personnel du héros en tant que chrétien et Scandinave, mais aussi à la définition des marges du monde scandinave et des populations qui la composent. Par un effet de miroir inversé, la magnification des qualités du héros entraîne ainsi la définition d'un idéal scandinave qui s'oppose aux populations rencontrées dans cet espace. La géographie physique, très lacunaire, fait alors place à une conception mentale de la géographie et des frontières reposant sur la définition de l'altérité et la prise de conscience de l'unité culturelle scandinave.

Ce phénomène fait en réalité écho au contexte politique dans lequel les sagas islandaises de la fin du XIII siècle et du début du XIVe siècle furent rédigées. Cette période correspond à la perte de l'autonomie de l'île entre 1262-1264, et débouche en termes littéraires sur la réévaluation du passé des insulaires et la redéfinition de leur identité. À travers des généalogies de rois et de héros liés directement à l'histoire de la Scandinavie, le genre des fornaldarsögur a tenté d'offrir un passé commun à l'ensemble des pays nordiques et par là même de se lier davantage à cet héritage. Le changement politique subi à la fin du XIII e siècle força l'aristocratie scandinave à réévaluer son passé et à le lier davantage à l'histoire et à la géographie du monde scandinave, aux dépens de racines considérées jusqu'alors comme exclusivement norvégiennes ${ }^{57}$. Ceci explique, semble-t-il, pourquoi la Rus', une terre éminemment liée à ce monde, tient une place si importante dans ce type de récits. À une époque d'affirmation des structures étatiques, les écrivains ont tenté de fournir un matériel permettant d'entériner la définition des frontières du monde scandinave à travers la définition de l'autre. Toutefois, ce phénomène pourrait aussi être le corollaire d'un changement du statut de l'État russe sur la scène internationale suite à la conquête mongole avec en point d'orgue en 1238 les prises de Vladimir et de Moscou, puis de Kiev en 1240. Il serait ainsi intéressant de mesurer l'impact que cette vague de conquête a eu dans les mentalités ouest-européennes et scandinaves au niveau de la production littéraire. Les contemporains, choqués par ce changement

55. Au contraire de récits qui concernent l'ouest ou l'Atlantique Nord où il est parfois question du nombre de jours de voyages.

56. L'Yngvars saga nous décrit qu'après avoir navigué plusieurs jours ils remarquèrent que «les animaux avaient d'autres habitudes et couleurs, ce dont ils déduisirent qu'ils s'éloignaient de leurs propres districts et pays», "Saga d'Yngvar le Grand Voyageur», 2009, chap. 5, p. 46.

57. SigurĐSSON, 2009, p. 59-78. 
du paysage duquel ils étaient pourtant familiers, ont pu lorsqu'il s'agissait de décrire la Russie d'un point de vue ethnographique, offrir un panorama teinté d'orientalisme dans un monde qu'ils considéraient comme n'appartenant plus au monde occidental, voire chrétien ${ }^{58}$.

\section{Bibliographie}

AHoLA, Joonas, «Outlaws, women and violence. In the social margins of saga literature», in Á austrvega, Saga and East Scandinavia, Preprint papers of the $14^{\text {th }}$ International Saga Conference, Uppsala $9^{\text {th }}-15^{\text {th }}$ August 2009, Agneta Ney, Henrik Williams et Fredrik Charpentier LjungQvist (éd.), Gävle, Gävle University Press, 2009, p. 21-28.

BOyer, Régis, «Vita - historia - saga. Athugun formgerðar», Gripla, 6, 1984.

BOYER, Régis, «Les sagas islandaises sont-elles des documents historiques?», Cahier des Annales de Normandie, 23, 1990, p. 109-126.

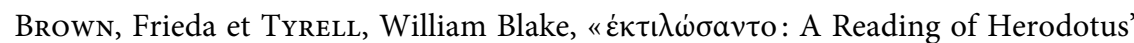
Amazons», The Classical Journal, 80, nº 4, 1985, p. 297-302.

Chronique de Nestor (récit des temps passés): naissance des mondes russes, traduite du vieux-russe par Jean-Pierre ARrignon, Toulouse - Marseille, Anacharsis, 2008.

GLAuser, Jürg, Isländische Märchensagas, Studien zur Prosaliteratur im spätmittelalterlichen Island, Bâle, Helbing und Lichtenhahn, 1983.

GodolPhin, Francis Richard Borroum, «Herodotus: On the Scythians», The Metropolitan Museum of Art Bulletin, 32, $\mathrm{n}^{\circ}$ 5, tiré de The Lands of the Scythians: Ancient Treasures from the Museums of the U.S.S.R 3000 B.C-10o B.C., 1973-1974, p. 129-149.

Hálfdanar saga Eysteinssonar, Franz Rolf SCHRÖDER (éd.), Altnordische Saga-Bibliothek, 15, Halle, Niemeyer, 1917.

Hauksbók, udgiven efter de arnamagnceanske håndskrifter, no. 371, 544 og 675, 4${ }^{\circ}$, samt forskellige papirshåndskrifter, EIRíkUR Jónsson et FINNUR Jónsson (éd.), Copenhagen, Thieles Boktrykkeri, 1892-1896.

Islandske Annaler, indtil 1578. Utgivne for det Norske historiske kildesfrikftfond, Gustav Storm (éd.), Khristiana, Grøndahl, 1888 (réimpr.: Oslo, 1977) (Den Norske Historiske Kildeskriftsfond shrifter, 2).

JACKson, Tatjana, «The North of Eastern Europe in Early Nordic Texts: the Study of Place-Names», Arkiv for Nordisk Filologi, 108, 1993, p. 38-45.

JACKson, Tatjana, «The Image of Old rus in Old Norse Literature (a place-name study) », Middelalderforum, Tverrfaglig Tidsskrift for Middelalderstudier, 1-2, 2003, p. $29-56$.

JACKson, Tatjana, "Ways on the "Mental Map" of Medieval Scandinavians", in Analecta Septentrionalia, Wilhelm Heizmann, Klaus Böldl et Heinrich BeCK (éd.), Berlin, New York, Walter de Gruyter, 2009, p. 211-220.

58. Sur les problèmes liés à la définition de l'Europe et du monde chrétien à l'époque médiévale, voir Oschema, 2012, p. 59-72. 
JACKson, Tatjana, «Icelandic skalds and Garðar», in Vers l'Orient et vers l'Occident. Regards croisés sur les dynamiques et les transferts culturels des Vikings à la Rous ancienne, Pierre BAuduin et Alexander Musin (éd.), Caen, Presses universitaires de Caen (Publications du Crahm), 2014, p. 45-53.

JACKson, Tatjana et Podossinov, Alexander, «Norway in Old Norse Literature: Some Considerations on the Specific Character of Scandinavia Spatial Orientation », Skandinavistik, 27-2, 1997, p. 85-97.

Jón ViĐar Sigurðsson, "Historical Writing and the Political Situation in Iceland 1100-1400", in Negotiating Pasts in the Nordic Countries, Interdisciplinary Studies in History and Memory, Anne Eriksen et Jón ViĐar Sigurðsson, Lund, Nordic Academic Press, 2009, p. 59-78.

Jungmann, Astrid, Monstrous Transformations in Old Icelandic Sagas, Mémoire de Master, Reykjavík, Icelandic and Comparative Cultural Studies, Medieval Icelandic Studies, Háskóli Íslands, September 2011.

Konungs annáll, Annales islandorum regii, isländska handskriften n:o 2087 4:to i den gamla samlingen på det stora kungliga biblioteket $i$ Köpenhamn i diplomatariskt avtrick utgiven, H. BUergel Goodwin (éd.), Uppsala, Akademiska Bokhandeln, 1906.

L'Edda poétique, Textes présentés, annotés et traduits par Régis Boyer, Paris, Fayard, 1974 (réimpr. 1992).

Lionarons, Joyce Tally, The Medieval Dragon, The Nature of the Beast in Germanic Literature, Enfield Lock, Hisarlik Press, 1998.

Lönnroth, Lars, From History to Legend: The Yngvar Stones and Yngvar saga viðförla, London, Viking Society, 2012, https://www.academia.edu/3694169/ Yngvars_2520saga.

«Lögmannsannáll», in Annálar og nafnaskrá, GuĐNi Jónsson (éd.), Reykjavík (2 ${ }^{\text {nd }}$ éd.), Íslendingasagnaútgáfan Haukadalsútgáfan, 1953, p. 75-158.

Marez, Alain, Anthologie Runique, Paris, Les Belles Lettres, 2007.

McLennan, Alistair, Monstrosity in Old English and Old Icelandic Literature, $\mathrm{PhD}$ Thesis, University of Glasgow, 2010.

Örvar-Odds saga, Richard Constant Boer (éd.), Leiden, Brill, 1888.

Oschema, Klaus, "Medieval Europe, Object and Ideology», in Ideas of/for Europe. An Interdisciplinary Approach to European Identity, Teresa Pinheiro, Beata Cieszynska et José Eduardo Franco (éd.), Frankfurt am Main, Peter Lang, 2012, p. 59-72.

Power, Rosemary, "Journey to the Otherworld in the Icelandic Fornaldarsögir ", Folklore, 96, $\mathrm{n}^{\circ}$ 2, 1985, p. 156-175.

Rómverja saga, PORBJörg Helgadóttir (éd.), Reykjavík, Stofnun Árna Magnússonar í Islenskum Fræðum, 2010.

«Saga d'Egill le Manchot et d'Ásmundr meurtrier des Berserkir», in Sagas légendaires islandaises, Textes présentés et traduits du vieil islandais par Régis BoyER avec le concours de Jean Renaud, Toulouse, Anacharsis, 2012, p. 979-1014.

«Saga de Hervör et du roi Heidrekr», in Sagas légendaires islandaises, Textes présentés et traduits du vieil islandais par Régis Boyer avec le concours de Jean RENAUd, Toulouse, Anacharsis, 2012, p. 115-176. 
Saga de Hrólfr sans Terre, Traduite du vieil islandais, présentée et annotée par Régis BOyer, Toulouse, Anacharsis, 2004.

«Saga d'Oddr aux Flèches», in Sagas légendaires islandaises, Textes présentés et traduits du vieil islandais par Régis Boyer avec le concours de Jean RenAud, Toulouse, Anacharsis, 2012, p. 829-946.

«Saga de Ragnarr aux Braies velues», in Sagas légendaires islandaises, Textes présentés et traduits du vieil islandais par Régis Boyer avec le concours de Jean Renaud, Toulouse, Anacharsis, 2012, p. 177-220.

«Saga des Völsungar», in Sagas légendaires islandaises, Textes présentés et traduits du vieil islandais par Régis Boyer avec le concours de Jean Renaud, Toulouse, Anacharsis, 2012, p. 31-114.

«Saga d'Yngvar le Grand Voyageur» in La Russie des Vikings, Saga d'Yngvar le Grand Voyageur suivie du Dit d'Eymundr Hringsson, Textes présentés, annotés et traduits par Régis Boyer, Toulouse, Anacharsis, 2009.

Schach, Paul, «Saga», in Medieval Scandinavia, An Encyclopedia, Phillip Pulsiano, Kirsten Wolf (éd.), New York, London, Garland Publishing, 1993, p. 561.

SCHÄFKE, Werner, "The "Wild East" in Late Medieval Icelandic Romances - Just a $\operatorname{Prop}(\mathrm{p})$ ? », in Á austrvega, Saga and East Scandinavia, Preprint papers of the $14^{\text {th }}$ International Saga Conference, Uppsala $9^{\text {th }}-15^{\text {th }}$ August 2009, Gävle, Gävle University Press, 2009, p. 845-850.

Schulz, Katja, Riesen, Von Wissenshutern und Wildnisbewohnern in Edda und Saga, Heidelberg, Universititsverlag Winter, 2004.

Shafer, John Douglas, Saga-Accounts of Norse Far-Travellers, Thèse de doctorat, Durham, Department of English Studies, Durham University, 2010, http://etheses. dur.ac.uk/286/.

SHEPARD, Jonathan, "The Rhôs guests of Louis the Pious: whence and wherefore?", Early Medieval Europe, 4, 1995, p. 41-60.

Simek, Rudolf, «Elusive Elysia, or which Way to Glæsisvellir?», in Sagnaskemmtum, Studies in honour of Hermann Pálsson, Rudolf SimEK, Jónas KRISTJÁNsson et Hans BeKker-Nielsen (éd.), Vienne, Hermann Böhlaus (Philologica Germanica, 8), 1986, p. $247-275$.

Simek, Rudolf, Heaven and Earth in the Middle Ages, The Physical World before Columbus, Woodbridge, Boydell and Brewer, 1996.

Snorri Sturluson, La Saga de Harald l'Impitoyable, Traduite du vieil islandais, présentée et annotée par Régis Boyer, Paris, Payot, 1979.

Snorri Sturluson, La Saga de saint Óláf, Traduite du vieil islandais, présentée et annotée par Régis Boyer, Paris, Payot, 1983 (réimpr. 2007).

StURla PórĐArson, «Hákonar saga Hákonarsonar», in Det Arnamagnoeanske håndskrift 81 a fol, (Skálholtsbók yngsta), Inneholdende: Sverris saga, Böglunga sögur, Hákonar saga Hákonarsonar, Albert KJÆR et Ludvig Holm-OlsEN (éd.), Kristiania, Oslo, Norsk Historisk Kjeldeskrift-Institutt, 1910.

The Etymologies of Isidore of Seville, Stephen BARney, Wendy Lewis, Jennifer BEACH et Oliver Berghof (éd.), Cambridge, Cambridge University Press, 2006.

Turville-Petre, Edward Oswald Gabriel, Scaldic poetry, Oxford, Clarendon Press, 1976. 
Zuckerman, Constantin, "Deux étapes de la formation de l'ancien État russe», in Les centres proto-urbains russes entre Scandinavie, Byzance et Orient, Michel Kazanski, Anne Nercessian et Constantin Zuckerman (éd.), Paris, Lethielleux, 2000, p. 95-120.

Джаксон, Татьяна Николаевна, «Sýrnes и Gaðar, загадки древнескандинавской топонимии Древней Руси ", Scando-Slavica, 32, 1986, p. 73-83.

Куза, Андрей Васильевич, «Русские раннесредневековые города , Тезисы докладов советской делегаиии на III Международном конгрессе славянской археологии, Братислава, сентябрь, 1975 год, Институт археологии АН СССР, Москва, Наука, 1975, р. 62-65.

Рыдзевская, Елена Александровна, «Рыдзевская, Елена Александровна, О названии Руси Garðaríki », Древняя Русь и Скандинавия в IX-XIV вв., материалы и исследования, Москва, Наука, 1978, р. 143-151.

Тихомиров, Михаил Николаевич, Основные труды: Древнерусские города, 2-е издание, Москва, Госполитиздат, 1956. 